\title{
Inositol 1,4,5-trisphosphate receptor type 3 is involved in resistance to apoptosis and maintenance of human hepatocellular carcinoma
}

\author{
MARCONE LOIOLA DOS SANTOS ${ }^{1,2}$, ANDRESSA FRANÇA ${ }^{2}$, ANTÔNIO CARLOS MELO LIMA FILHO ${ }^{2}$, \\ RODRIGO M. FLORENTINO ${ }^{2}$, PAULO HENRIQUE DINIZ ${ }^{3}$, FERNANDA OLIVEIRA LEMOS ${ }^{2}$, \\ CARLOS ALBERTO XAVIER GONÇALVES ${ }^{4}$, VITOR LIMA COELHO ${ }^{4}$, \\ CRISTIANO XAVIER LIMA ${ }^{5}$, GISELLE FOUREAUX ${ }^{1}$, MICHAEL H. NATHANSON ${ }^{6}$, \\ PAULA VIEIRA TEIXEIRA VIDIGAL ${ }^{7}$ and M. FÁTIMA LEITE ${ }^{2}$
}

\begin{abstract}
Departments of ${ }^{1}$ Morphology, ${ }^{2}$ Physiology and Biophysics and ${ }^{3}$ Internal Medicine, Federal University of Minas Gerais, Belo Horizonte, Minas Gerais 31270-901; ${ }^{4}$ Coordination of Biotechnology, SENAI's Innovation Institute for Biosynthetics and Fibers, SENAI CETIQT, Rio de Janeiro 20961-020; ${ }^{5}$ Department of Surgery, Federal University of Minas Gerais, Belo Horizonte, Minas Gerais 30 130-100, Brazil; ${ }^{6}$ Section of Digestive Diseases, Department of Internal Medicine, Yale University School of Medicine, New Haven, CT 06520-8056, USA; ${ }^{7}$ Department of Pathological Anatomy and Forensic Medicine of Hospital das Clínicas, Federal University of Minas Gerais, Belo Horizonte, Minas Gerais 30 130-100, Brazil
\end{abstract}

Received March 2, 2021; Accepted September 24, 2021

DOI: $10.3892 / \mathrm{ol} .2021 .13150$

\begin{abstract}
The expression of the inositol 1,4,5-trisphosphate receptor type 3 (ITRP3) in hepatocytes is a common event in the pathogenesis of hepatocellular carcinoma (HCC), regardless of the type of underlying liver disease. However, it is not

Correspondence to: Professor Paula Vieira Teixeira Vidigal, Department of Pathological Anatomy and Forensic Medicine of Hospital das Clínicas, Federal University of Minas Gerais, 3rd floor, Av. Alfredo Balena 190, Santa Efigênia, Belo Horizonte, Minas Gerais 30 130-100, Brazil

E-mail: pvidigal@medicina.ufmg.br
\end{abstract}

Abbreviations: AFP, $\alpha$ fetoprotein; ALD, alcoholic liver disease; CAAE, Certificado de Apresentação de Apreciação Ética (Certificate of Presentation of Ethical Appreciation); CC, cryptogenic cirrhosis; CHILD, CHILD-Pugh scores; CHOP, C/EBP homologous protein; CLD, chronic liver disease; CTSB, cathepsin B; EFS, event-free survival; H\&E, hematoxylin and eosin; HCC, hepatocellular carcinoma; $\mathrm{HCV}$, hepatitis $\mathrm{C}$ virus; HepG2, immortalized liver cancer cell line; HPF, high-power fields; INR, international normalized ratio; ITPR, inositol 1,4,5-trisphosphate receptor; ITPR1, inositol 1,4,5-trisphosphate type 1 receptor; ITPR2, inositol 1,4,5-trisphosphate type 2 receptor; ITPR3, inositol 1,4,5-trisphosphate type 3 receptor; MELD, model for end-stage liver disease; OLT, orthotopic liver transplantation; $\mathrm{PH}$, partial hepactomy; RNA-seq, RNA sequencing; TBIL, total bilirubin

Key words: apoptosis, calcium signaling, chronic diseases, liver cancer, mitosis known whether ITPR3 expression in hepatocytes is involved in tumor maintenance. The aim of the present study was to determine whether there is an association between ITPR3 expression and clinical and morphological parameters using HCC samples obtained from liver explants from patients $(n=53)$ with different etiologies of underlying chronic liver disease (CLD). ITPR3 expression, mitosis and apoptosis were analyzed in human liver samples by immunohistochemistry. Clinical and event-free survival data were combined to assess the relationship between ITPR3 and liver cancer growth in patients. RNA sequencing analysis was performed to identify apoptotic genes altered by ITPR3 expression in a liver tumor cell line. ITPR3 was highly expressed in HCC tumor cells relative to adjacent CLD tissue and healthy livers. There was an inverse correlation between ITPR 3 expression and mitotic and apoptotic indices in HCC, suggesting that ITPR3 contributed to the maintenance of HCC by promoting resistance to apoptosis. This was confirmed by the upregulation of CTSB, CHOP and GADD45, genes involved in the apoptotic pathway in HCC. The expression of ITPR3 in the liver may be a promising prognostic marker of $\mathrm{HCC}$

\section{Introduction}

Hepatocellular carcinoma (HCC) is the commonest primary liver malignancy and its incidence is increasing worldwide (1). According to the GLOBOCAN database, in 2020, including intrahepatic bile ducts, there were 905,677 new cases of liver cancer around the world, accompanied by 830,180 new deaths (2). Liver cirrhosis is a major risk factor for HCC (3). Although liver transplantation is the primary long term curative treatment, HCC still has a recurrence rate of $10-15 \%$ in 
transplant patients (4). Since biopsy is not mandatory for HCC diagnosis (5), the availability of tumor specimens for analysis is limited, which in turn has limited our understanding of the molecular mechanisms involved in carcinogenesis.

The calcium ion $\left(\mathrm{Ca}^{2+}\right)$ is an intracellular second messenger involved in energy metabolism, cell cycle control, gene expression, cell proliferation, cell migration, necrosis and apoptosis in nearly every cell type, including hepatocytes (6). The inositol 1,4,5-trisphosphate receptor (ITPR) is the only intracellular $\mathrm{Ca}^{2+}$ release channel expressed in the liver, with all three isoforms described in humans and rodents $(7,8)$. The expression of each ITPR varies among cell type, having distinct subcellular distribution and biophysical properties (9). For instance, under physiological conditions type 1 (ITPR1) and 2 (ITPR2) are the predominant isoforms in hepatocytes $(10,11)$, while isoform 3 (ITPR3) is absent or minimally expressed (12). However, ITPR 3 becomes significantly expressed in hepatocytes in acute $(13,14)$ and chronic liver diseases, as well as in HCC and in liver cancer cell lines $(15,16)$. ITPR 3 expression is also increased in other malignancies, including cholangiocarcinoma (17), colon cancer (18), melanoma (19), mesothelioma and prostate cancer (20).

Epigenetic events have been associated with the expression of ITPR3 in HCC, with hypomethylation of the promoter region of the ITPR3 gene upregulating its expression (16). In contrast, the promoter regions of ITPR 1 and ITPR 2 are already demethylated in normal hepatocytes (16), consistent with the observation that ITPR 1 and ITPR 2 are constitutively expressed in this cell type (10).

To understand the biological role of ITPR3 in HCC, the present study compared ITPR3 expression in HCC and adjacent cirrhotic parenchyma in patients with different types of underlying chronic liver disease. It also correlated ITPR3 expression with clinical and morphological parameters in order to investigate whether ITPR3 could serve as a prognostic marker.

\section{Materials and methods}

Sample characterization. A total of 53 liver explants from patients who underwent orthotopic liver transplantation (OLT) in Hospital das Clínicas, Universidade Federal de Minas Gerais (UFMG; Federal University of Minas Gerais), Brazil, between January 2002 and December 2017, were retrospectively reviewed after the study was approval by the local Ethics Committee, COEP-UFMG, in the city of Belo Horizonte, state of Minas Gerais, Brazil (CAAE 71206617.8.0000.5149) and written informed consent was obtained from the patients or their relatives. Histologically normal tissues were obtained from liver resections of patients with metastatic colon cancer between 2010 and 2017 at Hospital das Clínicas (UFMG), after rigorous examination by an experienced pathologist.

A representative slide of each case was selected and analyzed after staining with conventional hematoxylin and eosin (H\&E) and immunohistochemistry. Formalin-fixed (concentration 10\%; duration $12 \mathrm{~h}$ at room temperature) and paraffin-embedded samples were deparaffinized and antigen retrieval was performed in citrate buffer $(10 \mathrm{mM})$ containing $0.6 \%$ peroxide of hydrogen (heating temperature, $65^{\circ} \mathrm{C}$ for $8 \mathrm{~h}$ in the thermal oven), followed by four immersions in xylene (the first for $20 \mathrm{~min}$ and the others with $1 \mathrm{~min}$ duration) and three immersions in absolute alcohol (the first for $5 \mathrm{~min}$ and the others with 1 min duration). Afterwards, the slides were immersed in running water for $3 \mathrm{~min}$. The Novolink Polymer Detection System (cat. no. RE7200-CE; Leica Microsystems, Inc.) was used in the subsequent steps as described previously (21). Primary antibodies against ITPR3 (anti-ITPR3; Sigma-Aldrich; Merck KGaA; cat. no. HPA003915) and ssDNA labeling (anti-Single Stranded DNA; cat. no. 18731; Immuno-Biological Laboratories, Co., Ltd.) were incubated overnight at room temperature in a 1:100 dilution, followed by incubation with detection polymer for $40 \mathrm{~min}$ at room temperature. DAB was used for signal detection.

Clinical pre-OLT data and histopathological parameters of the explanted liver were retrospectively collected from the medical records, including sex, age, etiology of the underlying chronic liver disease (CLD), model for end-stage liver disease (MELD) and CHILD-Pugh scores (CHILD); prognostic models that estimate the severity of underlying liver condition, pre-OLT serum levels of $\alpha$ fetoprotein (AFP), number of nodules, size of the greatest nodules, microvascular invasion and histological grade. The medians of MELD, AFP, total bilirubin, international normalized ratio, creatinine, alanine aminotransferase, aspartate aminotransferase and serum albumin were obtained from the sum of the values of each of these clinical data for each patient. Therefore, the medians reflect the mean of the total sum of the 53 patients in this study, between the minimum and maximum values.

Outcomes, such as death from any cause or tumor recurrence since OLT and event-free survival (EFS), defined as the time interval between the OLT date and the occurrence of the event or ending of the follow-up period (December 20, 2017), were analyzed.

Analysis of ITPR3 expression intensity and ssDNA labeling index. To evaluate ITPR 3 expression, images of immunohistochemistry slides stained for ITPR 3 from 10 different fields (magnification, $\mathrm{x} 40$ ) were captured in tumor and adjacent cirrhotic parenchyma through an optical microscope (Zeiss $\mathrm{GmbH})$. The intensity of ITPR3 staining was evaluated by delimiting 10 cytoplasmic regions, excluding the nucleus, using ImageJ 1.50i software (National Institutes of Health). The software performed this analysis through a histogram, which expressed the intensity of the pixels in each selected region on a scale between 0-255. ITPR3 expression intensity was expressed as the mean of 10 analyzed cells in each of the 10 imaged fields.

Mitotic index evaluation. A total of two experienced liver pathologists identified areas of interest and counted the number of mitotic cells in 10 high-power fields (HPF) of H\&E stained slides (magnification, x20) in order to identify areas containing mitotic figures, in metaphase or anaphase. Following Baak (22), the mitotic cells were counted only with a complete agreement between the two pathologists through a multi-head microscope. The delineation between low mitotic index in samples with $>5$ mitotic figures at $10 \mathrm{HPF}$ and high mitotic index in samples with $\geq 5$ mitotic figures at $10 \mathrm{HPF}$ was based on previous studies (23-27). 
Pre-processing of raw RNA sequencing (RNA-seq) data. Raw read sequences were pre-processed by filtering out read sequences with less than $36 \mathrm{bp}$, removing low quality or $\mathrm{N}$ bases from the read ends, trimming Illumina adapters, scanning the read with a 4-base wide sliding window and cutting when the average quality per base dropped below 15 (LEADING:3 TRAILING:3 SLIDINGWINDOW:4:15 MINLEN:36) by Trimmomatic v0.39 (28).

The raw RNA-seq data were obtained in our previous publication, through experiments with HepG2 (16), considered a cell line of liver cancer. A commercially available CRISPR/Cas9 system was used to eliminate ITPR3 in HepG2 liver cancer cells (Santa Cruz Biotechnology, Inc.). Cells were grown in Dulbecco's Modified Eagles Medium (cat. no. 11320033) supplemented with L-glutamine (1 mM; cat. no. 25030149), fetal bovine serum (10\% v/v; cat. no. A4766801) and penicillin/streptomycin (100 units/ml and $100 \mathrm{mg} / \mathrm{ml}$; cat. no. 15140122) at $37^{\circ} \mathrm{C}$ in a humid environment with an artificial atmosphere containing $5 \% \mathrm{CO}_{2}$.

Quantification and differential gene expression analysis. Quantification of the gene expression (-g) was performed by Salmon v1.2.1 (29). The transcriptome index was built using the cDNA sequences of the Homo sapiens assembly version GRCh38 downloaded from Ensembl release 104 (30). The estimated number of reads were used for the differential gene expression analysis. Genes with low expression levels were removed; genes with counts scaled by total mapped reads per million $>1$ in at $\geq 9$ samples were retained for differential gene expression analysis. DESeq2 (31) was applied to evaluate the differential gene expression using DEBrowser v1.16.1 (32). Genes were considered differentially expressed when they had an adjusted P-value <0.05; fold-change $\leq-1.5$ (downregulated or more enriched in control condition).

Identification of differentially expressed genes associated with apoptosis. Genes participating in the human apoptosis route were extracted from the Kyoto Encyclopedia of Genes and Genomes (KEGG) Pathways (33) Apoptosis map (hsa04210) was obtained using the rest application programming interface of KEGG. Genes on the apoptosis pathway were mapped to their corresponding UniProt identifiers by UniProt's idmapping (The Uniprot Consortium 2021) (34). The same was done for the ensemble gene IDs of differentially expressed genes. An in-house custom python script was used to compare both lists and identify differentially expressed genes participating on the apoptosis pathway. A custom colorized version of the apoptosis pathway, containing upregulated genes in green, downregulated genes in red and non-differentially expressed genes in white was generated using KEGG Mapper (35).

Heatmap. Heatmaps of the top 50 differentially expressed genes sorted by the average Reads per Kilobase per Million of mapped reads were generated for each condition by Heatmapper (36) using average linkage clustering and Euclidean measurement methods.

Statistical analysis. Descriptive statistics summarized the data, which were expressed as numbers and percentages (categorical) and as median and interquartile range (continuous). A normality test (Shapiro-Wilk) was performed for each continuous variable. For comparison between the etiological groups, $\chi^{2}$ or the Fisher's exact test was applied in categorical variables and Mann Whitney or Kruskal-Wallis test in continuous data. In multiple comparisons, Dunn's test was applied after the Kruskal-Wallis test. Multivariate COX regression model with covariance structure was performed to determine the model for EFS. Moreover, to evaluate the correlation between the clinicopathological parameter and ITPR 3 expression, Spearman's coefficient was used. SPSS software, v20 (IBM Corp.), was used for statistical analysis. Three technical replicates were used per sample in the analyses. Final results were expressed as the mean of the three values. $\mathrm{P}<0.05$ was considered to indicate a statistically significant difference.

\section{Results}

The etiologies of the underlying chronic liver diseases among the explanted livers were distributed as follows: Hepatitis $\mathrm{C}$ virus $(\mathrm{HCV})$ infection $(\mathrm{n}=31 ; 58.5 \%)$, alcoholic liver disease (ALD; $n=16 ; 30.2 \%$ ) and cryptogenic cirrhosis $(C C ; n=6$; $11.3 \%)$. Multiple etiologies or coexistence of other liver pathologies were considered exclusion criteria.

Among all etiological groups, the median age of the patients was 57.09 years (range, 41.11-74.03). The median MELD score was 15.0 (range, 6.0-32.0) and the median AFP was 123.78 (range, 1.00-2883.00). The median total bilirubin was 2.65 (range, 0.50-8.70), the median international normalized ratio was 1.045 (range, 0.990-2.690) and the median creatinine was 1.02 (range, 0.14-3.00). The median alanine aminotransferase was 91.3 (range, 24.0-325.0), the median aspartate aminotransferase was 85.54 (range, 25.00-267.00) and the median serum albumin was 3.20 (range, 2.00-4.80). All clinical and laboratory data are summarized in Table I.

Morphological descriptions of the livers from HCC patients are presented in Table II. The number of nodules was categorized into two groups: $\leq 3$ nodules and $>3$ nodules. Vascular invasion was categorized as present or absent. Differentiation grade, according to Edmondson and Steiner (37), was classified into I and II (Low grade; well differentiated) and III and IV (high grade; poorly differentiated). The non-categorical data (mitosis and nodule size) are expressed as median values.

As ITPR 3 expression is a common event in HCC development (16), the presence and distribution of this $\mathrm{Ca}^{2+}$ channel was evaluated in HCC samples from different etiologies. ITPR3 staining of cholangiocytes was used as an internal positive control, as this liver cell type constitutively expresses ITPR 3 (29). There was greater ITPR 3 labeling, both in the non-tumor regions of the explants (Fig. 1A) and the tumor regions (Fig. 1B), relative to hepatocytes from healthy control livers. ITPR 3 labeling in the tumor region of patients with HCC caused by HCV (Fig. 1C) or ALD (Fig. 1D) were higher in comparison with the adjacent non-tumor region, while no difference was observed between these two regions in CC patients (Fig. 1E). Although the level of ITPR3 staining was similar among the etiologic groups in both the cirrhotic parenchyma and the tumor regions (Fig. 1F), the pattern of labelling varied among groups. In the tumor regions and in the underlying cirrhotic parenchyma, homogeneous staining of ITPR3 was observed, with ITPR 3 dispersed throughout the 
Table I. Clinical and laboratory data of patients with hepatocellular carcinoma.

\begin{tabular}{lccc}
\hline Parameter & Minimum & Median & Maximum \\
\hline Age (years) & 41.11 & 57.09 & 74.03 \\
MELD & 6.0 & 15.0 & 32.0 \\
AFP (ng/ml) & 1.00 & 123.78 & 2883.00 \\
TBIL (mg/dl) & 0.50 & 2.65 & 8.70 \\
INR & 0.990 & 1.045 & 2.690 \\
Creatinine (mg/dl) & 0.14 & 1.02 & 3.00 \\
ALT (U/l) & 24.0 & 91.3 & 325.0 \\
AST (U/l) & 25.00 & 85.54 & 267.00 \\
ALB (g/dl) & 2.00 & 3.20 & 4.80 \\
\hline
\end{tabular}

MELD, model for end-stage liver disease; AFP $\alpha$ fetoprotein; TBIL, total bilirubin; INR, international normalized ratio; ALT, alanine aminotransferase; AST, aspartate transaminase; ALB, albumin.

Table II. Anatomopathological characteristics of liver from patients with hepatocellular carcinoma.

\begin{tabular}{lc}
\hline Clinical parameters & Value \\
\hline Nodules number & \\
$\leq 3$ & \\
$>3$ & $26(55.3 \%)$ \\
Vascular invasion $^{\mathrm{a}}$ & $21(44.7 \%)$ \\
Present & \\
Absent & $23(54.8 \%)$ \\
Differentiation degree & \\
Low degree (I-II) $^{\mathrm{a}}$ & $19(45.2 \%)$ \\
High degree (III-IV) & \\
Histological type & \\
Pseudo-acinar & $35(72.9 \%)$ \\
Solid & $13(27.1 \%)$ \\
Trabecular & \\
Pseudo-acinar/trabecular $_{\text {Mitosis (10 HPF) }}^{\mathrm{b}}$ & $6(12.8 \%)$ \\
Nodule size (cm) $^{\mathrm{b}}$ & $3(6.4 \%)$ \\
\end{tabular}

Data are expressed as absolute numbers (percentage) or ${ }^{\mathrm{b}}$ median (interquartile range). HPF, high-power fields.

hepatocyte cytoplasm (Fig. 1A and B; blue arrows). In some cases, as observed in $\mathrm{CC}$, a predominant perinuclear region of ITPR3 staining (Fig. 1A and B; black arrows) was present.

As in other malignancies, ITPR3 expression is an early event in HCC carcinogenesis $(16,18)$ which may then relate to morphological aspects as well as clinical outcomes of patients affected by the disease. Thus, the correlation between ITPR3 expression and clinicopathological parameters was investigated. Table III showed that serum AST levels positively correlated with the intensity of the tumor ITPR3 labeling profile $(\mathrm{P}<0.02)$, a correlation that was not observed in the
Table III. Correlation between ITPR3 labeling intensity with anatomopathological and clinical data parameters.

\begin{tabular}{lcc}
\hline $\begin{array}{l}\text { Clinical } \\
\text { parameters }\end{array}$ & $\begin{array}{c}\text { Cirrhosis } \\
\text { P-value }\end{array}$ & $\begin{array}{c}\text { Tumor } \\
\text { P-value }\end{array}$ \\
\hline Number nodules & 0.120 & 0.296 \\
Vascular invasion & 0.637 & 0.746 \\
Necrosis & 0.114 & 0.461 \\
Fibrosis & 0.752 & 0.456 \\
Tumor inflammation & 0.383 & 0.552 \\
Intracellular & 0.927 & 0.825 \\
characteristics & & \\
Differentiation & 0.677 & 0.668 \\
degree & & \\
Histological pattern & 0.601 & 0.941 \\
MELD & 0.395 & 0.393 \\
AFP (ng/ml) & 0.703 & 0.781 \\
TBIL (mg/dl) & 0.496 & 0.694 \\
INR & 0.403 & 0.344 \\
Creatinine (mg/dl) & 0.216 & 0.248 \\
ALT (U/l) & 0.410 & 0.281 \\
AST (U/l) & 0.497 & $0.018^{\mathrm{a}}$ \\
Mitosis (10 HPF) & - & $0.0098^{\mathrm{b}}$ \\
\hline
\end{tabular}

MELD, model for end-stage liver disease; AFP, $\alpha$ fetoprotein; TBIL, total bilirubin; INR, international normalized ratio; ALT, alanine aminotransferase ( $\left({ }^{\mathrm{P}}<0.098\right.$. Spearman post hoc test); AST, aspartate transaminase; ALB, albumin; HPF, high-power fields $\left({ }^{\mathrm{b}} \mathrm{P}<0.098\right.$; Student t-test).

adjacent parenchyma ( $\mathrm{P}>0.4$; using Spearman's statistical test).

A high mitotic index ( $\geq 5 / 10 \mathrm{HPF}$ ) is a poor prognostic factor in HCC, as well as with intrahepatic metastasis, larger tumor size, higher AFP levels, advanced tumor stage $(24,28)$, vascular invasion, poor histological differentiation and recurrence rate (26). Therefore, the mitotic index was correlated with the intensity of ITPR3 labeling. Mitotic index was categorized as either low $(<5)$ or high $(>5)$. The majority of cases $(62.8 \%)$ had a low mitotic index $(n=22)$, while $37.2 \%(n=13)$ exhibited high mitotic index. In addition, patients with greater ITPR3 staining exhibited a lower mitotic index, while patients with less ITPR3 labeling intensity exhibited a higher mitotic index (Fig. 2A). The mitotic index was inversely associated with ITPR3 expression in the tumor region $(\mathrm{P}<0.01$; using unpaired Student's t-test). These results suggest that cell proliferation is not the process by which ITPR 3 regulates liver tumor maintenance. Therefore it was investigated whether $\mathrm{Ca}^{2+}$ may instead modulate apoptosis.

Apoptosis is involved in both cancer growth (19) and liver regeneration (30). Our group has previously demonstrated that ITPR3 expression can induce resistance to cell death in HCC (16). Thus, the correlation between the apoptotic marker ssDNA labelling and ITPR3 expression was investigated. In the HCC samples, 69.7\% $(n=23)$ had a low apoptotic index $(\leq 50 \%)$, whereas $30.3 \%(n=10)$ exhibited a high apoptotic percentage $(>50 \%)$ (Fig. 2B). Patients with higher intensity of 

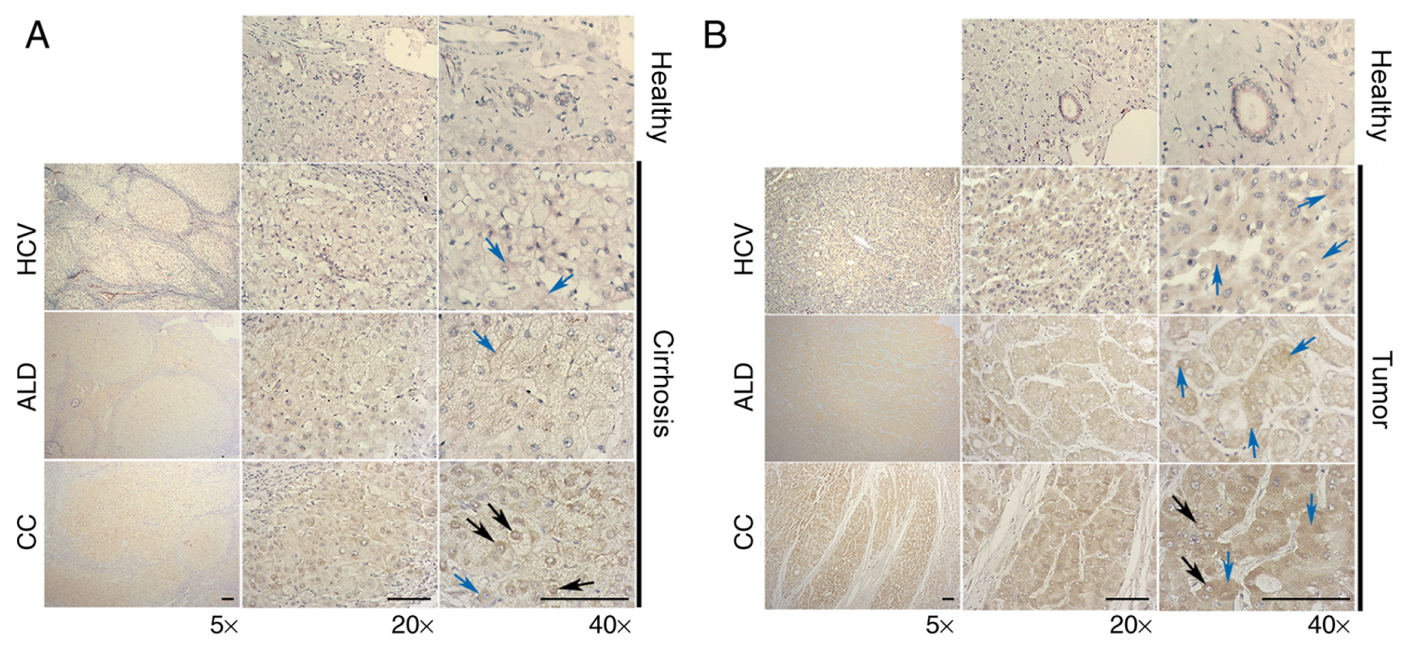

C
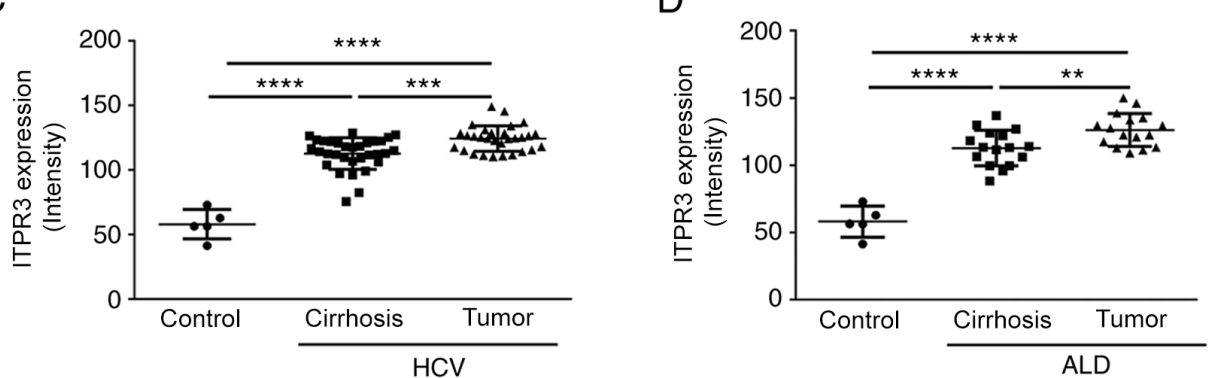

$\mathrm{E}$
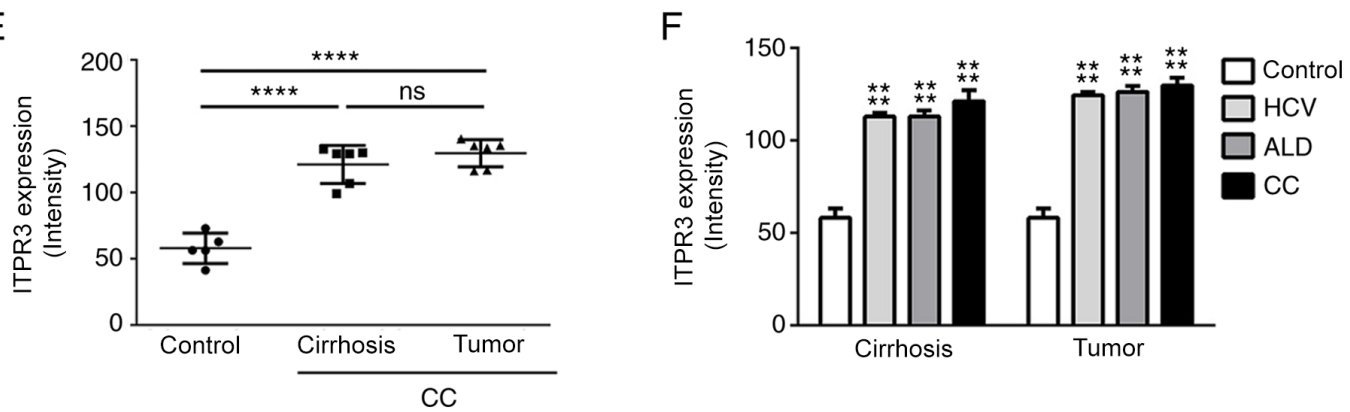

Figure 1. Representative images of ITPR3 labeling in HCC, underlying cirrhotic parenchyma and non-tumor livers (controls). (A) ITPR3 labeling in cirrhosis in patients diagnosed with HCV ( $\mathrm{n}=31)$, ALD $(\mathrm{n}=16), \mathrm{CC}(\mathrm{n}=6)$ and healthy (control; $\mathrm{n}=2)$. Scale bar=200 $\mu \mathrm{m}(\mathrm{magnification}, \mathrm{x} 5), 50 \mu \mathrm{m}(\mathrm{magnification}, \mathrm{x} 20)$ and $25 \mu \mathrm{m}$ (magnification, $\mathrm{x} 40$ ). Black arrows indicate ITPR3 perinuclear localization in hepatocytes. Blue arrows indicate ITPR3 cytoplasmatic localization in hepatocytes. (B) ITPR3 labeling in tumor (HCC) region of patients diagnosed with HCV ( $n=31)$, ALD ( $n=16)$, CC ( $n=6)$ and healthy (control; $n=3)$. Black arrows indicate ITPR3 perinuclear localization in hepatocytes. Blue arrows indicate ITPR3 cytoplasmatic localization in hepatocytes. (C) Representative graph of the intensity of ITPR 3 marking in HCC regions and cirrhotic parenchyma of patients with HCV compared with non-tumor livers (control; $n=31$; ${ }^{* * * * *} \mathrm{P}<0.0001$; $\left.{ }^{* * *} \mathrm{P}<0.0001\right)$. (D) Representative graph the intensity of ITPR3 marking in HCC regions and cirrhotic parenchyma of patients with ALD compared with non-tumor livers (control; $\mathrm{n}=16 ;{ }^{* *} \mathrm{P}<0.0057 ;{ }^{*}{ }^{* *} \mathrm{P}<0.0001$ ). (E) Representative graph the intensity of ITPR3 marking in HCC regions and cirrhotic parenchyma of patients with CC compared with non-tumor livers (control; $\mathrm{n}=06 ;{ }^{* * * *} \mathrm{P}<0.0001$ ). (F) Representative graph the ITPR3 labeling intensity in tumor samples and cirrhotic parenchyma comparing the three etiologies ( $\left.{ }^{* * * *} \mathrm{P}<0.0001\right)$. ITPR3, inositol 1,4,5-trisphosphate type 3 receptor; HCC, hepatocellular carcinoma; HCV, hepatitis C virus; ALD, alcoholic liver disease; CC, cryptogenic cirrhosis; ALD, alcoholic liver disease.

ITPR3 staining exhibited a low apoptotic percentage, while patients with lower intensity of ITPR3 displayed a higher apoptotic percentage $(\mathrm{P}<0.0005$; Fig. $2 \mathrm{C})$. These findings suggested that upregulation of ITPR3 correlates with higher apoptosis resistance, which could contribute to tumor maintenance.

As the mitotic index and apoptotic percentage were both inversely associated with the intensity of tumor ITPR3 staining, whether these two parameters correlated with each other in HCC was evaluated. In the specimens of the present study, the degree of apoptosis and mitosis were directly related $(\mathrm{P}<0.05$ by unpaired Student's t-test; Fig. 2D), indicating that these events were closely related in the context of the role of ITPR 3 in the modulation of the cell cycle and tumoral maintenance in HCC.
To improved understanding of the mechanism by which ITPR3 expression in hepatocytes is protective, rather than permissive, of cell death by apoptosis, RNA-seq was analyzed comparing global gene expression patterns between wild-type and ITPR3 knockout HepG2 cells. The apoptotic genes CTSB, CHOP and GADD45 were found to be upregulated in the ITPR 3 KO cells (Figs. 3 and S1). CTSB is a protease whose overexpression indicates poor prognosis of patients with $\mathrm{HCC}$, facilitating tumor migration and invasion (38). CHOP is a potential pro-apoptotic oncogene in $\mathrm{HCC}$, participating in the promotion of hepatic carcinogenesis through pathways involved in crosstalk with ATF6 (39). The GADD45 family of proteins responds to physiological and environmental cellular 
A

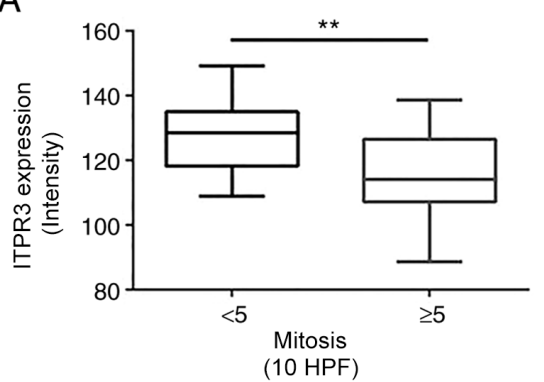

C

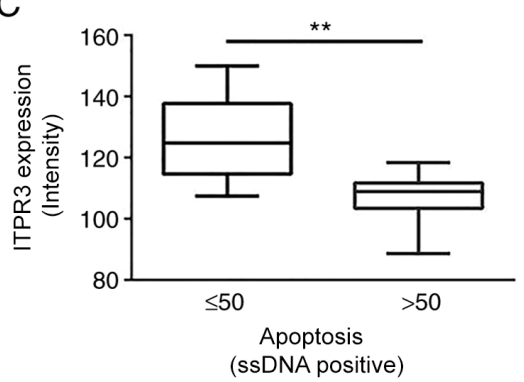

B

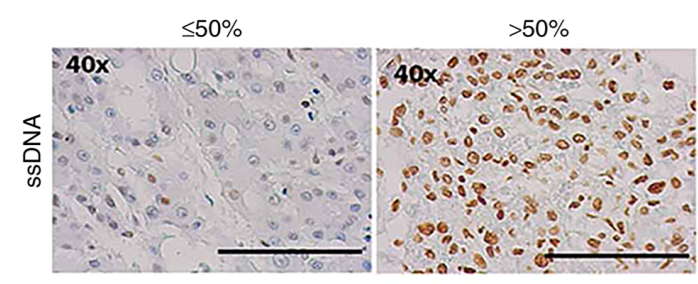

D

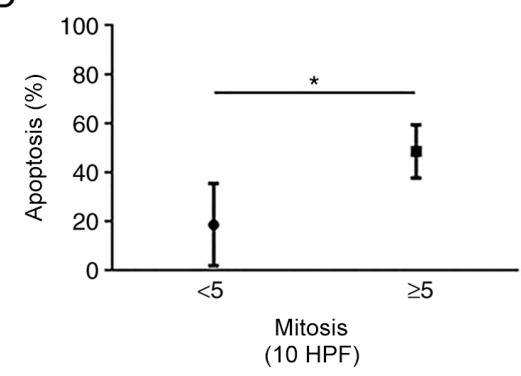

Figure 2. Correlation between ITPR3 labeling intensity and mitotic and apoptosis percentages (ssDNA). (A) Low mitotic $(<5)$, high mitotic $(\geq 5)$; $n=35$. ${ }^{* *} \mathrm{P}<0.0098$ Student's t-test. (B) Representative images of ssDNA labeling by immunohistochemistry, low nuclear labeling for ssDNA ( $\left.\leq 50\right) \%$ and high ssDNA labeling $(>50 \%)$; Scale bar $=25 \mu \mathrm{m}$ (magnification, $\mathrm{x} 40$ ). (C) Correlation graph between ITPR3 labeling intensity and apoptosis percentage. Low apoptosis percentage $(\leq 50 \%)$, high apoptosis percentage $(>50 \%) ; \mathrm{n}=33$. ${ }^{* *} \mathrm{P}<0.0003$ Student's t-test. (D) Correlation graph between mitotic index and percentage of apoptosis $\left(\mathrm{n}=15\right.$; $\left.{ }^{\mathrm{P}} \mathrm{P}<0.0220\right)$. ITPR3, inositol 1,4,5-trisphosphate type 3 receptor; ssDNA, single-stranded DNA; HPF, high-power fields.

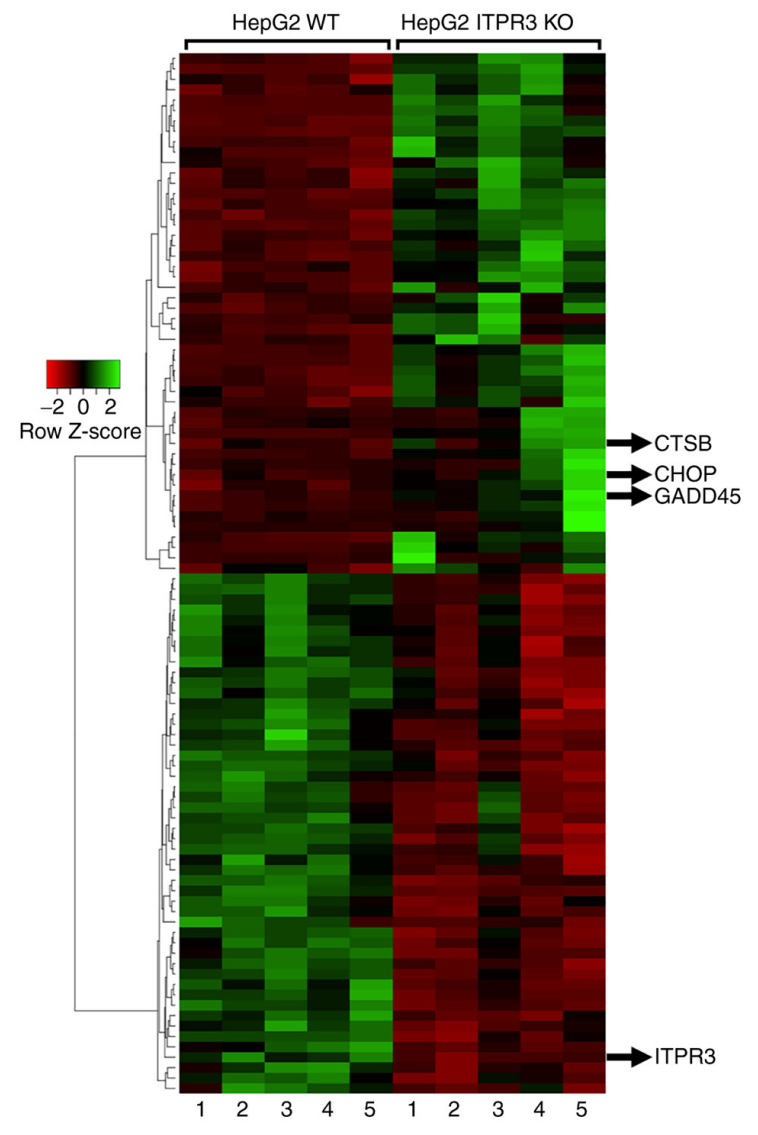

Figure 3. Heatmap of the most upregulated and downregulated genes in WT and ITPR 3 KO HepG2 cells. Five different samples were analyzed by RNA-seq and several genes were altered when ITPR3 was not expressed. To confirm efficient depletion of ITPR3 in these cells, the ITPR3 gene was highlighted between the downregulated genes. CTSB, CHOP and GADD45 were the genes associated with apoptosis. WT, wild type; KO, knockout; ITPR3, inositol 1,4,5-trisphosphate type 3 receptor.

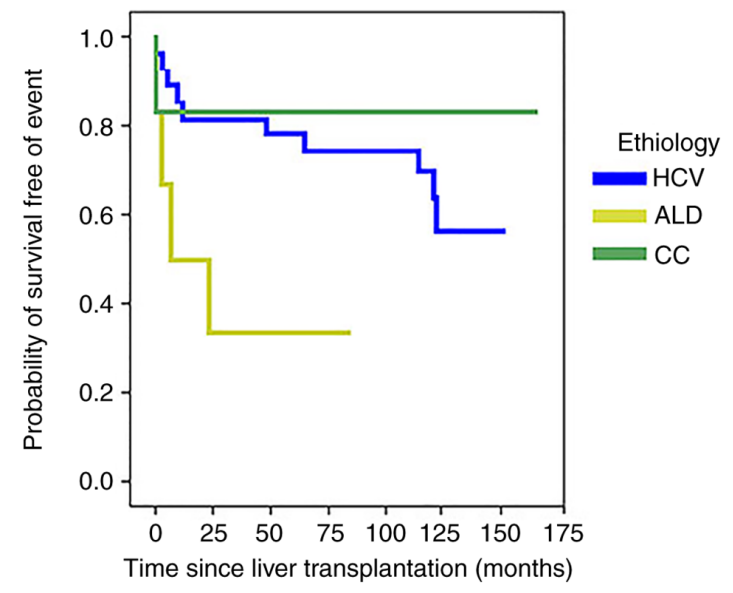

Figure 4. Analysis of the EFS among the three etiological groups. Kaplan-Meier curve, with a difference in the comparison of the EFS between the etiological groups (Renyi test=0.015). Blue line representing the EFS of patients with HCV-associated tumor; Green line representing the EFS of patients with CC-associated tumor and yellow line representing the EFS of patients with ALD-associated tumor. EFS, event-free survival; HCV, hepatitis $\mathrm{C}$ virus; $\mathrm{CC}$, cryptogenic cirrhosis; $\mathrm{ALD}$, alcoholic liver disease.

stress (40) through apoptosis (41), with a specific under-expression in HCC that correlates with worse prognosis (41). These results suggest that chronic ITPR 3 expression may promote an anti-apoptotic response pathway in liver cancer through modulation of gene expression and proteasome activity.

In the survival analysis, the median event-free survival (EFS) was 113.2 months (range, 93.1-133.2), considering all etiologies together. The Kaplan-Meier curve showed a significant difference comparing the EFS among etiologic groups (Renyi test=0.015; Fig. 4). This difference was identified 
between $\mathrm{CC}$ and $\mathrm{HCV}$ etiologies $(\mathrm{P}=0.001)$. However, neither ITPR 3 expression in the tumor nor in the cirrhotic region was associated with EFS outcome. Together, the results suggested that ITPR 3 expression in transformed hepatocytes is implicated in evasion of apoptosis, which contributes to the maintenance of $\mathrm{HCC}$.

\section{Discussion}

Hepatocarcinogenesis is a process that involves multiple steps and in which several pathways can interact (42). Among them, regulators of cell proliferation and survival are prominent (43). Although different types of underlying CLD can lead to tumorigenesis through different mechanisms $(44,45)$, de novo expression of the ITPR $3 \mathrm{Ca}^{2+}$ channel was noted as a common molecular signaling pathway that occurs in all HCC-related etiologies (16). The present study confirmed the involvement of ITPR3 in HCC of patients with HCV, ALD and CC.

Together, two main molecular classes of HCC can be identified (46). The proliferation class, characterized by a higher availability of signals involved in cell cycle proliferation and cell cycle progression, as well as progenitor cell markers, is associated with a more aggressive clinical course. Such tumors have higher AFP levels, less cell differentiation and more frequent vascular invasion $(47,48)$. These characteristics are in turn associated with greater chances of recurrence after initial treatment and lower survival rates $(48,49)$. HCC from hepatitis B virus infection is mostly in this class (50). The non-proliferative class of HCC possesses at least two central molecular characteristics: A predominance of Wnt signaling in $\leq 25 \%$ of cases and an immune response in the remainder of cases; in the physiological context, the tumor transcriptome of this class is similar to normal liver physiology (50). From a clinical perspective, tumors in this class exhibit less aggressive characteristics, such as higher cell differentiation index and lower AFP levels. Regarding the underlying etiology, HCV and ALD-related HCC are more frequent in this class (48).

In the sample of the present study, the main CLD etiologies were HCV and ALD, with average AFP serum levels of $123.8 \mathrm{ng} / \mathrm{ml}$, which is relatively low, as only levels above $400 \mathrm{ng} / \mathrm{ml}$ are considered as a threshold in the diagnosis of HCC (51). Regarding morphology, the tumor was well differentiated (grade I or II of Edmondson and Steiner score) in $72.9 \%$ of patients, the median nodule size was $<3.0 \mathrm{~cm}$ and $62.8 \%$ of tumors had a low mitotic index. Taken together, these data indicated that the majority of the tumors in the present study would be classified as non-proliferative. Moreover, the tumor stage was not as advanced, which is compatible with some methods of treatment received; OLT is considered for patients fulfilling the Milan Criteria, as in our hospital: Hospital das Clínicas of the Federal University of Minas Gerais.

The prognostic importance of the mitotic index and tumor stage is appreciated in a number of malignancies $(52,53)$. In addition, its value is similar to that of techniques such as nuclear antigen of cell proliferation (54) or DNA analysis $(55,56)$. In the context of HCC, there is evidence that a high mitotic index is an unfavorable prognostic indicator $(24,25,57,58)$, as well as an independent predictor of short-term survival in patients who undergo hepatectomy with curative intent (23). In addition, a high mitotic index ( $\geq 5 / 10 \mathrm{HPF}$ is associated with intrahepatic metastasis, larger tumor size, higher AFP levels and advanced tumor stage $(23,27)$, as well as vascular invasion (26), poor histological differentiation and high recurrence rate (23). In the samples in the present study, most patients (62.8\%) had a low mitotic index ( $<5 / 10 \mathrm{HPF})$. This corroborated in vivo and in vitro studies which demonstrated that both $\mathrm{HCV}$ and ALD negatively affect cell cycle, promoting a $\mathrm{G} 2 / \mathrm{M}$ phase arrest in HCC cell lines $(59,60)$.

Demethylation of the promoter of the ITPR 3 gene is necessary for its expression in hepatocytes and has been associated with cell proliferation in mouse livers after partial hepatectomy (PH) (16). The data of the present study showed that the higher the expression of ITPR3, the lower the mitotic index. These apparent discrepancies can be explained, in part, by the methodologies used in these studies (PCNA vs. mitotic index), as well as the type of samples analyzed (animal PH model vs. human HCC specimen). PCNA, used in the previous study, is known to serve an important role in the metabolism of nucleic acids and its main function is associated with DNA replication (61). However, PCNA may also be present in repair by DNA excision, cell cycle control, chromatin assembly and RNA transcription (61). Therefore, PCNA staining might also reflect cells in phases preceding mitosis. Another important factor is the type of sample used, either the PH model, in which hepatocytes are prompted to proliferate as a physiological process of the liver (62), or the liver with already established HCC. So, while previous work showed that ITPR 3 stimulates hepatocyte proliferation and that its sustained expression in hepatocytes might lead to liver cancer (16), the findings of the current study supported a role for ITPR 3 in the maintenance of the liver tumor by preventing cell death, rather than through enhanced hepatocyte proliferation.

It is known that liver growth involves apoptosis, both in physiological conditions, such as in regeneration (29) and in pathological situations, such as in HCC (16). Additionally, $\mathrm{Ca}^{2+}$ signaling through ITPR 3 is part of apoptotic cell death (30) and the role of ITPR 3 in carcinogenesis has been recently reviewed (63). ITPR 3 can participate in both inhibiting and promoting apoptosis, depending on the type of tumor (16). The present study demonstrated an inverse correlation between apoptosis and liver tumor ITPR 3 expression. This is consistent with a previous finding that ITPR3 triggers anti-apoptotic gene expression in HCC cell lines (16). Similarities occur in colon cancer cells, in which ITPR 3 overexpression is associated with decreased apoptosis (18) and in hepatocytes infected with yellow fever virus, in which higher ITPR3 hepatocyte expression prevents cell death through apoptosis (14).

ITPR3 expression can induce resistance to cell death in liver cancer by increasing the expression of POU4F1 and decreasing SIAH2 in WT cells compared with HepG2 ITPR3KO (16). The present study extended these previous observations using RNA-seq and observed that more genes have their expression profile altered in relation to ITPR 3 and, also, it is suggested that the signaling pathways associated with apoptosis resistance were altered in HepG2 ITPR3 KO cells (Fig. S1). In particular, a significant upregulation of CTSB, CHOP and GADD45 was observed, genes associated with either cell survival or apoptosis. 
In the context of HCC, CHOP is pro-apoptotic (39), performing a pro-tumorigenic function, being an oncogene in hepatic carcinogenesis (39). CHOP is ubiquitously expressed at very low levels normally, but is strongly expressed in cells subjected to severe stress (64). However, activation of this gene is not universally observed in human HCC, which leads to the suggestion that there are other oncogenic pathways that promote hepatocarcinogenesis independently of CHOP. Thus, this gene may participate more in the progression of $\mathrm{HCC}$ rather than in its induction (39). The data from the present study suggested that the action of ITPR 3 on CHOP expression was associated with the apoptotic signaling pathway by promoting maintenance of liver cancer development.

Growth arrest and damage to DNA 45 (GADD45) is a family composed of three homologous acidic proteins $(\alpha, \beta$ and $\gamma)(65)$, which respond to cellular stresses of environmental and physiological origin (41). GADD45 proteins are also involved in hepatic tumorigenesis, in both carcinogenesis and established HCC (65). In the context of HCC, GADD $45 \beta$ has a pro-apoptotic action and occurs at a low or absent expression level in neoplastic cells, a specific characteristic of this type of tumor (41). This is critically correlated with fundamental clinical-pathological characteristics of tumor development (41). The synergistic effect of aspirin and sorafenib treatment induces an increase in GADD $45 \beta$ in HCC, promoting apoptosis and control of tumor growth (66), an opposite effect of growth in the non-neoplastic liver (65), reflecting the opposing effects of this gene depending on the biological context. GADD $45 \beta$ responses reflect its dynamics in rapid adaptations (65), being a possible diagnostic and selection biomarker for the treatment of HCC (66). These data suggest that the appearance of ITPR 3 in liver cancer may promote tumor maintenance through the suppressive effect of GADD45 expression, especially of the $\beta$ isoform.

The present study demonstrated the upregulation of important genes involved in the apoptotic pathway in liver cancer, such as GADD45 and CHOP. However, high-throughput technology would add stronger evidence. Thus, the absence of microarray experiments is a limitation of the present study.

In other types of neoplasms or liver diseases, ITPR 3 can assume several roles. In prostate cancer, the mechanisms that promote the degradation of ITPR 3 and prevent apoptosis have been elucidated (19). In ischemia-reperfusion injury, ITPR3 serves a protective role in which expression of the protein serves to change the mechanism of hepatocyte death from necrosis to apoptosis (15). This demonstrates the versatility that ITPR3 assumes within the varied contexts of different diseases and organs, but all associated with apoptotic events. It has been demonstrated that ITPR 3 serves a role in proliferative stimulation and apoptosis block in liver cell lines and normal mouse liver (16). The current study provided evidence in human HCC samples that ITPR 3 serves a role in hepatocarcinogenesis by modulating apoptosis. Additional work will be needed to better understand the mechanism by which de novo expression of ITPR 3 correlates with mitosis and apoptosis in the pathogenesis of HCC, as well as whether this represents a potential target for therapy.

In conclusion, ITPR 3 was highly expressed in HCC tumor cells relative to its expression level in the underlying CLD and healthy livers. ITPR 3 expression was inversely correlated with apoptotic and mitotic indices in HCC, suggesting that ITPR3 contributed to the maintenance of $\mathrm{HCC}$, promoting resistance to apoptosis. This resistance to apoptosis and alteration of tumor cell survival occurred, at least in part, through an intracellular signaling pathway in which ITPR 3 demonstrated a negative effect on the expression of CHOP and GADD45, providing a reduction in apoptotic cell death. The expression of ITPR 3 in the liver may be a promising prognostic marker of $\mathrm{HCC}$ and other types of liver cancer.

\section{Acknowledgements}

Not applicable.

\section{Funding}

The present study was supported by grants from CNPq, FAPEMIG and NIH (grant nos. P01-DK57751, P30-DK34989, R01-DK114041 and R01-DK112797).

\section{Availability of data and materials}

The datasets analyzed during the current study are available in the NCBI Sequence Read Archive under the Accession code PRJNA758563 (https://www.ncbi.nlm.nih. gov/sra/?term=PRJNA758563). All data generated or analyzed during this study are included in this published article.

\section{Authors' contributions}

The entire project was conducted, written and analyzed by MLDS, including carrying out the experiments. AF contributed in all aspects, mainly in the analysis and interpretation of data. RMF contributed with the assistance in carrying out the experiments, analysis and discussion of data and with the preparation of graphics and images. ACMLF contributed with the assistance in carrying out the experiments, analysis and discussion of data and with the preparation of graphics and images. PHD contributed to the statistical analysis and in the organization of the survival data. CAXG and VLC elaborated all the bioinformatics data, analysis and RNA-seq results. PVTV performed the mitotic cell count on the HCC samples in the tumor mitotic index experiment. In addition, PVTV helped to analyse and interpret the data in conjunction with the literature already published and was involved in the writing of the manuscript and in the critical review of important intellectual content. PVTV also helped with the construction of the data discussion. MFL participated in carrying out the experiments and articulating the data obtained from the works previously published by the group. FOL was involved in the writing of the manuscript and critical review of important intellectual content, and also contributed to the analysis of some of the data presented. MHN contributed to the analysis and discussion of the data in this study, in addition to approving the final version submitted for publication. GF participated in the orientation and design of the study, particularly in the contribution of immunohistochemical staining image analysis. CXL participated in the acquisition of clinical data from patients and in the interpretation of these data in conjunction with the results. MLDS and PVTV confirm the authenticity of all the raw data. All authors reviewed and approved the final manuscript. 


\section{Ethics approval and consent to participate}

The present study was approved by the Ethics Committee of the Hospital das Clínicas, Federal University of Minas Gerais (approval number CAAE 71206617.8.0000.5149) and written informed consent was obtained from the patients or their relatives, including for use of their tissue in the research.

\section{Patient consent for publication}

Not applicable.

\section{Competing interests}

The authors declare that they have no competing interests.

\section{References}

1. Agni RM: Diagnostic histopathology of hepatocellular carcinoma: A case-based review. Semin Diagn Pathol 34: 126-137, 2017.

2. Sung H, Ferlay J, Siegel RL, Laversanne M, Soerjomataram I, Jemal A and Bray F: Global cancer statistics 2020: GLOBOCAN estimates of incidence and mortality worldwide for 36 cancers in 185 countries. CA Cancer J Clin 71: 209-249, 2021.

3. Forner A, Reig M and Bruix J: Hepatocellular carcinoma. Lancet 391: 1301-1314, 2018

4. Bodzin AS, Lunsford KE, Markovic D, Harlander-Locke MP Busuttil RW and Agopian VG: Predicting mortality in patients developing recurrent hepatocellular carcinoma after liver transplantation: Impact of treatment modality and recurrence characteristics. Ann Surg 266: 118-125, 2017.

5. Villanueva A: Hepatocellular carcinoma. N Engl J Med 380: $1450-1462,2019$

6. Amaya MJ and Nathanson MH: Calcium signaling in the liver. Compr Physiol 3: 515-539, 2013.

7. Patel S, Joseph SK and Thomas AP: Molecular properties of inositol 1,4,5-trisphosphate receptors. Cell Calcium 25: 247-264, 1999.

8. Wojcikiewicz RJ: Type I, II, and III inositol 1,4,5-trisphosphate receptors are unequally susceptible to down-regulation and are expressed in markedly different proportions in different cell types. J Biol Chem 270: 11678-11683, 1995.

9. Yule DI, Ernst SA, Ohnishi H and Wojcikiewicz RJ: Evidence that zymogen granules are not a physiologically relevant calcium pool Defining the distribution of inositol 1,4,5-trisphosphate receptors in pancreatic acinar cells. J Biol Chem 272: 9093-9098, 1997.

10. Cruz LN, Guerra MT, Kruglov E, Mennone A, Garcia CR, Chen J and Nathanson MH: Regulation of multidrug resistance-associated protein 2 by calcium signaling in mouse liver. Hepatology 52: 327-337, 2010.

11. Hirata K, Pusl T, O'Neill AF, Dranoff JA and Nathanson MH: The type II inositol 1,4,5-trisphosphate receptor can trigger $\mathrm{Ca}^{2+}$ waves in rat hepatocytes. Gastroenterology 122: 1088-1100, 2002.

12. Dufour JF, Lüthi M, Forestier M and Magnino F: Expression of inositol 1,4,5-trisphosphate receptor isoforms in rat cirrhosis Hepatology 30: 1018-1026, 1999.

13. Lemos FO, França A, Lima Filho ACM, Florentino RM, Santos ML, Missiaggia DG, Rodrigues GOL, Dias FF, Souza Passos IB, Teixeira MM, et al: Molecular mechanism for protection against liver failure in human yellow fever infection. Hepatol Commun 4: 657-669, 2020.

14. Lima Filho ACM, França A, Florentino RM, Dos Santos ML de Oliveira Lemos F, Missiaggia DG, Fonseca RC, Gustavo Oliveira A, Ananthanarayanan M, Guerra MT, et al: Inositol 1,4,5-trisphosphate receptor type 3 plays a protective role in hepatocytes during hepatic ischemia-reperfusion injury. Cell Calcium 91: 102264, 2020.

15. Leite MF, Thrower EC, Echevarria W, Koulen P, Hirata K, Bennett AM, Ehrlich BE and Nathanson MH: Nuclear and cytosolic calcium are regulated independently. Proc Natl Acad Sci USA 100: 2975-2980, 2003.

16. Guerra MT, Florentino RM, França A, Lima Filho AC, Dos Santos ML, Fonseca RC, Lemos FO, Fonseca MC, Kruglov E, Mennone A, et al: Expression of the type $3 \mathrm{InsP}_{3}$ receptor is a final common event in the development of hepatocellular carcinoma. Gut 68: 1676-1687, 2019.
17. Ueasilamongkol P, Khamphaya T, Guerra MT, Rodrigues MA, Gomes DA, Kong Y, Wei W, Jain D, Trampert DC, Ananthanarayanan M, et al: Type 3 inositol 1,4,5-trisphosphate receptor is increased and enhances malignant properties in cholangiocarcinoma. Hepatology 71: 583-599, 2020.

18. Shibao K, Fiedler MJ,Nagata J, Minagawa N, Hirata K, Nakayama Y, Iwakiri Y, Nathanson MH and Yamaguchi K: The type III inositol 1,4,5-trisphosphate receptor is associated with aggressiveness of colorectal carcinoma. Cell Calcium 48: 315-323, 2010.

19. Kuchay S, Giorgi C, Simoneschi D, Pagan J, Missiroli S, Saraf A, Florens L, Washburn MP, Collazo-Lorduy A, Castillo-Martin M, et al: PTEN counteracts FBXL2 to promote IP3R3 - and $\mathrm{Ca}^{2+}$-mediated apoptosis limiting tumour growth. Nature 546: 554-558, 2017.

20. Bononi A, Giorgi C, Patergnani S, Larson D, Verbruggen K, Tanji M, Pellegrini L, Signorato V, Olivetto F, Pastorino S, et al: BAP1 regulates IP3R3-mediated $\mathrm{Ca}^{2+}$ flux to mitochondria suppressing cell transformation. Nature 546: 549-553, 2017.

21. Fonseca MC, França A, Florentino RM, Fonseca RC, Lima Filho AC, Vidigal PT, Oliveira AG, Dubuquoy L, Nathanson MH and Leite MF: Cholesterol-enriched membrane microdomains are needed for insulin signaling and proliferation in hepatic cells. Am J Physiol Gastrointest Liver Physiol 315: G80-G94, 2018.

22. Baak JP: Mitosis counting in tumors. Hum Pathol 21: 683-685, 1990.

23. Ha SY, Choi M, Lee T and Park CK: The prognostic role of mitotic index in hepatocellular carcinoma patients after curative hepatectomy. Cancer Res Treat 48: 180-189, 2016.

24. Haratake J, Takeda S, Kasai T, Nakano S and Tokui N: Predictable factors for estimating prognosis of patients after resection of hepatocellular carcinoma. Cancer 72: 1178-1183, 1993.

25. Nanashima A, Tanaka K, Yamaguchi H, Shibasaki S, Morino S, Yoshinaga M, Sawai T, Nakagoe T and Ayabe H: Fibrosis and inflammatory activity in noncancerous tissue and mitotic index of cancer tissue in patients with hepatocellular carcinoma: Relationship to clinicopathological factors and prognosis after hepatic resection. Dig Dis Sci 48: 1517-1522, 2003.

26. Osório FM, Vidigal PV, Ferrari TC, Lima AS, Lauar GM and Couto CA: Histologic grade and mitotic index as predictors of microvascular invasion in hepatocellular carcinoma. Exp Clin Transplant 13: 421-425, 2015.

27. OuchiK, Sugawara T, Ono H,Fujiya T, Kamiyama Y,Kakugawa Y, Mikuni J, Yamanami H, Komatsu S and Horikoshi A: Mitotic index is the best predictive factor for survival of patients with resected hepatocellular carcinoma. Dig Surg 17: 42-48, 2000.

28. Bolger AM, Lohse M and Usadel B: Trimmomatic: A flexible trimmer for illumina sequence data. Bioinformatics 30 : 2114-2120, 2014.

29. Patro R, Duggal G, Love MI, Irizarry RA and Kingsford C: Salmon provides fast and bias-aware quantification of transcript expression. Nat Methods 14: 417-419, 2017.

30. Howe KL, Achuthan P, Allen J, Allen J, Alvarez-Jarreta J, Amode MR, Armean IM, Azov AG, Bennett R, Bhai J et al: Ensembl 2021. Nucleic Acids Res 49D: D884-D891, 2021.

31. Love MI, Huber W and Anders S: Moderated estimation of fold change and dispersion for RNA-seq data with DESeq2. Genome Biol 15: 550, 2014

32. Kucukural A, Yukselen O, Ozata DM, Moore MJ and Garber M: DEBrowser: Interactive differential expression analysis and visualization tool for count data. BMC Genomics 20: 6, 2019.

33. Kanehisa M and Goto S: KEGG: Kyoto encyclopedia of genes and genomes. Nucleic Acids Res 28: 27-30, 2000.

34. UniProt Consortium: UniProt: The universal protein knowledgebase in 2021. Nucleic Acids Res 49D: D480-D489, 2021.

35. Kanehisa $M$ and Sato Y: KEGG Mapper for inferring cellular functions from protein sequences. Protein Sci 29: 28-35, 2020.

36. Babicki S, Arndt D, Marcu A, Liang Y, Grant JR, Maciejewski A and Wishart DS: Heatmapper: Web-enabled heat mapping for all. Nucleic Acids Res 44W: W147-W153, 2016.

37. Edmondson HA and Steiner PE: Primary carcinoma of the liver: A study of 100 cases among 48,900 necropsies. Cancer 7: 462-503, 1954.

38. Ruan J, Zheng H, Rong X, Rong X, Zhang J, Fang W, Zhao P and Luo R: Over-expression of cathepsin B in hepatocellular carcinomas predicts poor prognosis of HCC patients. Mol Cancer 15: 17, 2016.

39. Scaiewicz V, Nahmias A, Chung RT, Mueller T, Tirosh B and Shibolet O: CCAAT/enhancer-binding protein homologous (CHOP) protein promotes carcinogenesis in the DEN-induced Hepatocellular carcinoma model. PLoS One 8: e81065, 2013. 
40. Liebermann DA and Hoffman B: Gadd45 in stress signaling. J Mol Signal 3: 15, 2008.

41. Qiu W, David D, Zhou B, Chu PG, Zhang B, Wu M, Xiao J, Han T, Zhu Z, Wang T, et al: Down-regulation of growth arrest DNA damage-inducible gene 45beta expression is associated with human hepatocellular carcinoma. Am J Pathol 162: 1961-1974, 2003.

42. Llovet JM, Peña CE, Lathia CD, Shan M, Meinhardt $G$ and Bruix J; SHARP Investigators Study Group: Plasma biomarkers as predictors of outcome in patients with advanced hepatocellular carcinoma. Clin Cancer Res 18: 2290-2300, 2012.

43. Alves RC, Alves D, Guz B, Matos C, Viana M, Harriz M, Terrabuio D, Kondo M, Gampel O and Polletti P: Advanced hepatocellular carcinoma. Review of targeted molecular drugs. Ann Hepatol 10: 21-27, 2011.

44. Ho DW, Lo RC, Chan LK and Ng IO: Molecular pathogenesis of hepatocellular carcinoma. Liver Cancer 5: 290-302, 2016.

45. Lu LC, Hsu CH, Hsu C and Cheng AL: Tumor heterogeneity in hepatocellular carcinoma: Facing the challenges. Liver Cancer 5: 128-138, 2016.

46. Hoshida Y, Nijman SM, Kobayashi M, Chan JA, Brunet JP, Chiang DY, Villanueva A, Newell P, Ikeda K, Hashimoto M, et al: Integrative transcriptome analysis reveals common molecular subclasses of human hepatocellular carcinoma. Cancer Res 69: 7385-7392, 2009.

47. Chiang DY, Villanueva A, Hoshida Y, Peix J, Newell P, Minguez B, LeBlanc AC, Donovan DJ, Thung SN, Solé M, et al Focal gains of VEGFA and molecular classification of hepatocellular carcinoma. Cancer Res 68: 6779-6788, 2008.

48. Villanueva A, Hoshida Y, Battiston C, Tovar V, Sia D, Alsinet C, Cornella H, Liberzon A, Kobayashi M, Kumada H, et al: Combining clinical, pathology, and gene expression data to predict recurrence of hepatocellular carcinoma. Gastroenterology 140: 1501-1512.e2, 2011.

49. Lee JS, Chu IS, Heo J, Calvisi DF, Sun Z, Roskams T, Durnez A, Demetris AJ and Thorgeirsson SS: Classification and prediction of survival in hepatocellular carcinoma by gene expression profiling. Hepatology 40: 667-676, 2004.

50. Zucman-Rossi J, Villanueva A, Nault JC and Llovet JM: Genetic landscape and biomarkers of hepatocellular carcinoma. Gastroenterology 149: 1226-1239.e4, 2015.

51. Masuzaki R and Omata M: Screening program in high-risk populations. In: Hepatocellular Carcinoma. 2nd edition. Mcmasters KM and Vauthey JN (eds). Springer, New York, NY, pp55-68, 2011.

52. Bloom HJ and Richardson WW: Histological grading and prognosis in breast cancer; a study of 1409 cases of which 359 have been followed for 15 years. Br J Cancer 11: 359-377, 1957.

53. Vang R, Shih IM and Kurman RJ: Ovarian low-grade and high-grade serous carcinoma: Pathogenesis, clinicopathologic and molecular biologic features, and diagnostic problems. Adv Anat Patht 16: 267-282, 2009.

54. Suehiro T, Matsumata T, Itasaka H, Yamamoto K, Kawahara N and Sugimachi K: Clinicopathologic features and prognosis of resected hepatocellular carcinomas of varied sizes with special reference to proliferating cell nuclear antigen. Cancer 76: 399-405, 1995.
55. Okada S, Shimada K, Yamamoto J, Takayama T, Kosuge T, Yamasaki S, Sakamoto M and Hirohashi S: Predictive factors for postoperative recurrence of hepatocellular carcinoma. Gastroenterology 106: 1618-1624, 1994.

56. Ruà $\mathrm{S}$, Comino A, Fruttero A, Torchio $\mathrm{P}$, Bouzari $\mathrm{H}$, Taraglio $\mathrm{S}$, Torchio B and Capussotti L: Flow cytometric DNA analysis of cirrhotic liver cells in patients with hepatocellular carcinoma can provide a new prognostic factor. Cancer 78: 1195-1202, 1996.

57. Soini Y, Virkajärvi N, Lehto VP and Pääkkö P: Hepatocellular carcinomas with a high proliferation index and a low degree of apoptosis and necrosis are associated with a shortened survival. Br J Cancer 73: 1025-1030, 1996.

58. Tannapfel A, Geissler F, Köckerling F, Katalinic A, Hauss J and Wittekind C: Apoptosis and proliferation in relation to histopathological variables and prognosis in hepatocellular carcinoma. J Pathol 187: 439-445, 1999.

59. Alisi A, Mele R, Spaziani A, Tavolaro S, Palescandolo E and Balsano C: Thr 446 phosphorylation of PKR by HCV core protein deregulates G2/M phase in HCC cells. J Cell Physiol 205: 25-31, 2005.

60. Clemens DL, Calisto LE, Sorrell MF and Tuma DJ: Ethanol metabolism results in a $\mathrm{G} 2 / \mathrm{M}$ cell-cycle arrest in recombinant Hep G2 cells. Hepatology 38: 385-393, 2003.

61. Juríková M, Danihel L, Polák Š and Varga I: Ki67, PCNA, and MCM proteins: Markers of proliferation in the diagnosis of breast cancer. Acta Histochem 118: 544-552, 2016.

62. Michalopoulos GK: Liver regeneration after partial hepatectomy: Critical analysis of mechanistic dilemmas. Am J Pathol 176: 2-13, 2010.

63. Mangla A, Guerra MT and Nathanson MH: Type 3 inositol 1,4,5-trisphosphate receptor: A calcium channel for all seasons. Cell Calcium 85: 102132, 2020.

64. Chikka MR, McCabe DD, Tyra HM and Rutkowski DT: C/EBP homologous protein (CHOP) contributes to suppression of metabolic genes during endoplasmic reticulum stress in the liver. J Biol Chem 288: 4405-4415, 2013.

65. Tian J and Locker J: Gadd45 in the liver: Signal transduction and transcriptional mechanisms. In: Gadd45 Stress Sensor Genes. Liebermann DA and Hoffman B (eds). Springer, New York, NY, pp69-80, 2013.

66. Xia H, Lee KW, Chen J, Kong SN, Sekar K, Deivasigamani A, Seshachalam VP, Goh BKP, Ooi LL and Hui KM: Simultaneous silencing of ACSL4 and induction of GADD45B in hepatocellular carcinoma cells amplifies the synergistic therapeutic effect of aspirin and sorafenib. Cell Death Discov 3: 17058, 2017.

This work is licensed under a Creative Commons Attribution-NonCommercial-NoDerivatives 4.0 International (CC BY-NC-ND 4.0) License. 